\title{
Systemic therapy of New World cutaneous leishmaniasis: A case report and review article
}

\author{
Amir Abadir MD ${ }^{1}$, Ameen Patel MB FRCPC ${ }^{1}$, Shariq Haider MD FRCPC ${ }^{1,2}$
}

\begin{abstract}
A Abadir, A Patel, S Haider. Systemic therapy of New World cutaneous leishmaniasis: A case report and review article. Can J Infect Dis Med Microbiol 2010;21(2):e79-e83.

Cutaneous leishmaniasis is a disease endemic to Central and South America, Mexico and the Caribbean, and affects millions of people. As travel to these regions becomes more common, cutaneous leishmaniasis is becoming a disease of increasing importance in the developed world. However, disease recognition and access to appropriate therapy for cutaneous leishmaniasis remains a challenge in North America. The present article reports a case of cutaneous leishmaniasis in a Canadian man following a trip to Costa Rica. Species-specific diagnosis was confirmed by polymerase chain reaction analysis of a skin biopsy, which was positive for Leishmania panamensis. After failing a course of itraconazole, the patient was successfully treated with sodium stibogluconate, despite significant barriers to administering this therapy, and the paucity of data regarding its efficacy and tolerability. The pathophysiology, diagnosis and systemic treatment of cutaneous leishmaniasis, as well as its emerging presence in the developed world, are reviewed.
\end{abstract}

\section{La thérapie systémique de la leishmaniose cutanée du Nouveau monde : Rapport de cas et exposé de synthèse}

\begin{abstract}
La leishmaniose cutanée est une maladie endémique en Amérique centrale et en Amérique du Sud, au Mexique et dans les Antilles, et elle touche des millions de personnes. Puisque les voyages dans ces régions deviennent plus fréquents, la leishmaniose gagne en importance dans les pays industrialisés. Cependant, le dépistage de la maladie et l'accès à une thérapie convenable demeurent problématiques en Amérique du Nord. Le présent article décrit le rapport d'un cas de leishmaniose cutanée chez un homme canadien au retour d'un voyage au Costa Rica. Le diagnostic spécifique d'espèce a été confirmé par analyse de réaction en chaîne de la polymérase d'une biopsie cutanée, positive à la Leishmania panamensis. Après l'échec d'un traitement à l'itraconazole, le patient a réagi de manière positive au stibogluconate de sodium, malgré les obstacles importants à l'administration de ce traitement et le peu de données sur son efficacité et sa tolérabilité. La physiopathologie, le diagnostic et le traitement systémique de la leishmaniose cutanée, de même que sa présence émergente dans les pays industrialisés, font l'objet d'un exposé.
\end{abstract}

Key Words: Cutaneous leishmaniasis; Itraconazole; Leishmania panamensis; PCR; Stibogluconate

\begin{abstract}
I eishmaniasis, a spectrum of conditions caused by parasites Lof the Leishmania genus, is endemic to many equatorial countries. There are estimated to be 1.5 to two million new cases per year, with as many as 350 million people worldwide at risk of acquiring the disease (1). There exists a distinct geographical classification in which the disease caused by Leishmania species that is native to Latin America is often referred to as New World leishmaniasis. Travel to these regions by people from North America and Europe is becoming more common (2). According to Statistics Canada, three of the top 15 countries visited by Canadians each year are located in Latin America and the Carribean - Mexico, Cuba and the Dominican Republic. In 2007, Mexico was the second most visited country by Canadians, followed by Cuba in fifth place and the Dominican Republic in sixth place. There were a total of 2433 visits to these countries that year, with over $\$ 2.3$ billion dollars spent on tourism (3).

This increase in travel has coincided with similar increases in the number of cases of cutaneous leishmaniasis noted in returning travellers. Between 1995 and 2003, there was a 3.5-fold increase in travel from the United Kingdom to Latin America, which was associated with a 3.2-fold increase in the number of cases of cutaneous leishmaniasis (4). A similar trend was noted in the Netherlands between 1990 and 2000 (5). This highlights cutaneous leishmaniasis as an emerging and important infection in the western world that warrants awareness from primary care
\end{abstract}

\begin{abstract}
physicians, hospitalists, general internists and infectious disease specialists. Cutaneous leishmaniasis presents a diagnostic challenge to clinicians who may be unfamiliar with its manifestations, and the paucity of research for this 'neglected disease' necessitates treatment with drugs that have a narrow therapeutic index, with which many physicians may not be familiar (6). Moreover, access to appropriate antiparasitic therapy is difficult and the infrastructure to implement outpatient therapy may present a major obstacle in the treatment of such patients.
\end{abstract}

\section{CASE PRESENTATION}

A 71-year-old man presented with a lesion on his right arm, which erupted shortly after returning from a vacation in Costa Rica. The lesion began as a small area of erythema, which was neither painful nor pruritic, but had gradually increased in size. He reported no discharge from the lesion or systemic symptoms such as fevers, chills and rigors. He could not recall being bitten by any insects and had not been ill while in Costa Rica. Interestingly, although he had been in Costa Rica with his partner, she did not develop any lesions. He had gone hiking in forested regions without his partner on several occasions, had worn short-sleeved shirts and shorts while hiking, and had not used insect repellents. His medical history was significant for age-related macular degeneration. His only medication was a daily multivitamin. He reported no allergies, was a lifelong nonsmoker, and denied alcohol and intravenous drug abuse.

\footnotetext{
${ }^{1}$ Department of Medicine; ${ }^{2}$ Division of Infectious Diseases, McMaster University, Hamilton, Ontario

Correspondence: Dr Shariq Haider, McMaster University Medical Centre, 1200 Main Street West, Room 3V51B, Hamilton, Ontario L8N 325.

Telephone 905-521-2100 ext 26287,fax 905-521-4971, e-mail haider@mcmaster.ca
} 


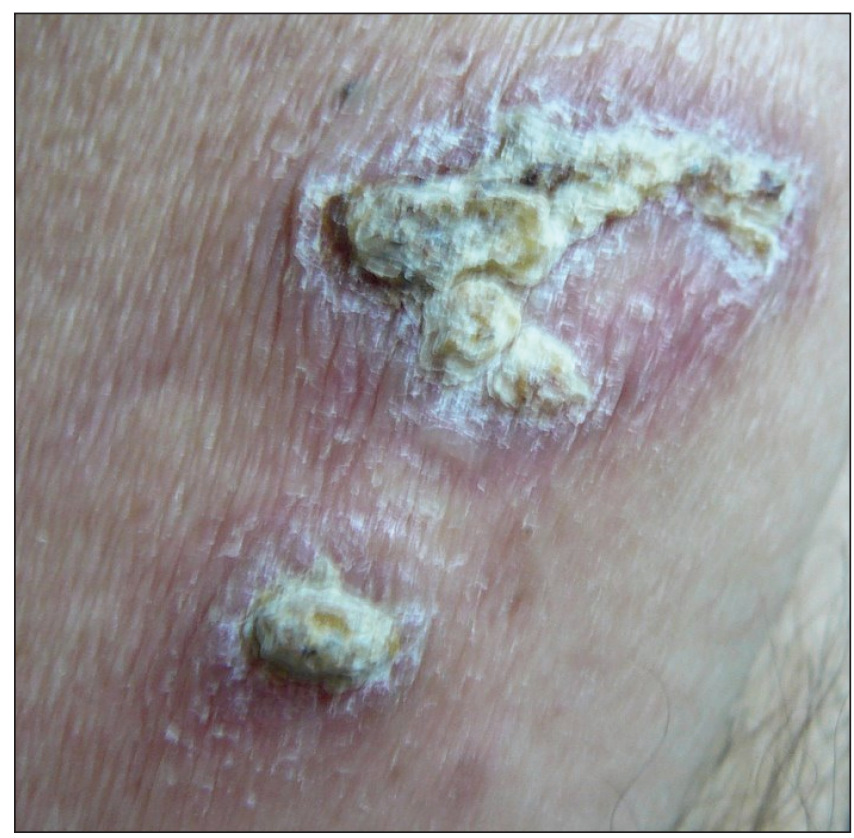

Figure 1) Cutaneous leishmaniasis of the right arm before treatment with intravenous sodium stibogluconate. Note the central raised areas with surrounding erythema and the presence of a satellite lesion

On examination, he was afebrile and his vital signs were normal. There was a $6 \mathrm{~cm}$ circumferential erythematous lesion with raised central areas located near the right axilla. A similar, but smaller, satellite lesion was identified adjacent to the larger lesion (Figure 1). There were no other lesions, rashes or palpable lymphadenopathy. His cardiac, respiratory and abdominal examinations were normal. Routine blood work including a complete blood count, electrolyte, renal, pancreatic enzyme and liver enzyme tests were normal. Biopsies of the lesion were sent to the National Reference Centre for Parasitology at McGill University (Montreal, Quebec), where histological evidence of amastigotes consistent with Leishmania species was noted. Polymerase chain reaction (PCR) analysis confirmed the presence of Leishmania panamensis (brasiliensis complex). The patient was initially treated with a $100 \mathrm{mg}$ course of oral itraconazole administered twice a day for three weeks without improvement, prompting referral to an infectious disease specialist $(\mathrm{SH})$ who recommended treatment with sodium stibogluconate.

The patient was admitted to hospital for treatment with sodium stibogluconate after approval was obtained through Health Canada's Special Access Program. He was monitored with remote cardiac telemetry and regular blood work for the first few days of treatment with intravenous sodium stibogluconate at a daily dose of $800 \mathrm{mg}$. The baseline 12-lead electrocardiogram (ECG), serial ECGs, telemetry and blood work were normal, with the exception of asymptomatic increases in serum lipase and transaminase levels of approximately twice the normal levels. There was difficulty in initiating outpatient therapy with intravenous sodium stibogluconate because the community care organizations were unfamiliar with the drug and were unwilling to administer it. Additionally, detailed communication and collaboration among physicians, nurses, the Community Care Access Centre (Hamilton, Ontario), the hospital pharmacy

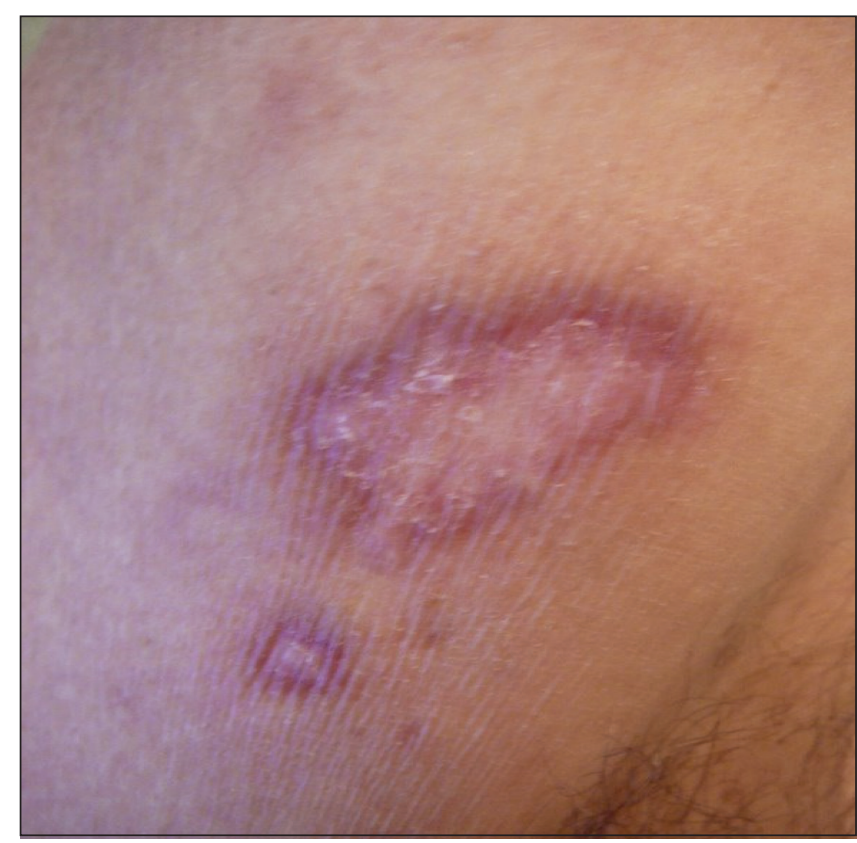

Figure 2) Cutaneous leishmaniasis of the right arm after 21 days of treatment with intravenous sodium stibogluconate. Note the resolution of the raised central areas, which is replaced with localized depigmentation

and the outpatient pharmacy was necessary to ensure that the drug was going to be available and administered properly. The patient was discharged four days after admission and received an additional 17 days of outpatient treatment. There was marked improvement in the lesion after the 21-day course of therapy (Figure 2).

\section{DISCUSSION}

Dermatological disorders are frequently seen in travellers returning from the developing world, and can be difficult to diagnose and treat, particularly among physicians in the developed world who are unlikely to be familiar with these conditions. Surveillance data acquired from nearly 18,000 travellers returning from countries in the developing world demonstrated that dermatological diseases accounted for 170 cases/1000 patients presenting to a travel medicine clinic (7). Among these patients, the majority were returning from the Caribbean and Latin America, which had an average of 251 cases of dermatological disease/1000 patients. Proportionally, among the 2947 patients returning from the developing world with a dermatological disease, leishmaniasis accounted for an average of 38 cases/1000 patients, with the highest rates observed in patients returning from Central and South America (64 cases/1000 patients and 143 cases/1000 patients, respectively). In fact, leishmaniasis was the most common dermatological disease seen in travellers returning from South America (7).

Leishmaniasis comprises a wide spectrum of conditions, all attributable to the protozoa of the genus Leishmania. Leishmaniasis is divided into cutaneous, mucosal and visceral disease. In New World (Latin American) leishmaniasis, the two most important subgenuses in human disease are Leishmania Mexicana complex and Leishmania Viannia (2). Within the Mexicana subgenus, L mexicana, Leishmania amazonensis and Leishmania venezuelensis may infect humans. Within the Viannia subgenus, Leishmania brasiliensis, L panamensis, 
Leishmania guyanensis and Leishmania peruviana may infect humans. Most of these species cause self-limited localized cutaneous leishmaniasis. However, $L$ brasiliensis and $L$ panamensis are also associated with destructive mucosal leishmaniasis, which can lead to facial disfigurement. None of these common Leishmania species are known to cause visceral leishmaniasis $(1,2)$.

In contrast with species of old world leishmaniasis (that is, southern Europe, Asia, Africa and the Middle East) that thrive in semi-arid environments, New World leishmaniasis species typically inhabit forested areas, and travel to these regions usually results in acquisition of the disease. Leishmaniasis is transmitted by the bite of the female sandfly, most commonly of the genus Lutzomyia in Latin America $(1,8)$. As the sandfly inserts its proboscis into the skin, the Leishmania promastigotes (flagellated forms) are introduced and are subsequently phagocytosed by macrophages. Within the macrophage, the promastigotes are enveloped within phagolysosomes and convert into amastigotes (unflagellated forms). On cell lysis, the amastigotes are released and infect other macrophages $(1,9)$. The cycle is repeated when the sandfly takes blood from an infected human. As infected macrophages are ingested by the sandfly, they are lysed and their amastigotes are released, which revert back to the promastigote stage in the gut and migrate toward the proboscis (1).

In localized cutaneous leishmaniasis, the lesion typically starts as an erythematous papule, which may be seen within a few days to many months, after initial inoculation of the parasite $(1,2)$. This subsequently progresses into an ulcer. Only rarely does the disease present as a predominantly nodular lesion (9). Lymphadenopathy, particularly with infections caused by L brasiliensis, may precede or accompany the development of cutaneous disease $(2,9)$. Despite this description, the actual appearance and time to heal of cutaneous leishmaniasis varies widely between different Leishmania species. Healing of the lesion may take as little as three to nine months for species such as L mexicana, or as long as six to 15 months for L brasiliensis and $L$ panamensis, after initial presentation (10). Although usually resulting in a permanent scar, a healed Leishmania lesion often imparts lifelong disease protection. The vast majority of patients with healed cutaneous leishmaniasis will have a positive leishmanin skin test (Montenegro test), but this may be negative during the first few months of active disease (1).

The differential diagnosis of leishmaniasis is wide and includes many conditions that are also commonly seen in endemic regions. Diseases that may mimic cutaneous leishmaniasis include bacterial skin infections, undifferentiated reactions to insect bites, leprosy, blastomycosis, cutaneous tuberculosis, syphilis, myiasis, impetigo, yaws, sarcoidosis and dermatological malignancies $(2,8)$.

The gold standard method for diagnosing cutaneous leishmaniasis is direct observation of the amastigotes in a Giemsastained biopsy specimen by light microscopy $(1,2,8)$. Typically, this requires the expertise of an experienced microbiologist who can differentiate between Leishmania parasites and similar organisms, such as Histoplasma species (8). Because light microscopy is relatively simple and inexpensive, it is the most widely used diagnostic method in endemic regions. In vitro culture is another common method of diagnosis and, unlike microscopy, allows for species-specific diagnosis via serological or isoenzyme analysis. However, culturing the parasite has poor sensitivity, is often time consuming and can be costly (1).

Given these limitations, PCR is now the diagnostic method of choice for cutaneous leishmaniasis in the developed world. It allows for rapid and species-specific diagnosis, and has an estimated sensitivity of between $89 \%$ and $100 \%$, and a specificity of essentially $100 \%(1,2)$. The amount of DNA required for amplification is minimal and is easily obtained from biopsies or scrapings of the lesion. Leishmania DNA has even been successfully amplified from blood samples of affected individuals (2). Unfortunately, many of the countries to which leishmaniasis is endemic lack the requisite facilities to perform PCRs (1). In cases of negative PCRs, in which clinical suspicion remains high, the histology of biopsy specimens can often be helpful.

Numerous drugs have been used to treat cutaneous leishmaniasis (1). The goals of treatment are, first and foremost, to prevent mucosal invasion and, second, to avoid disfiguring scars. Typically, one-third of patients will heal spontaneously, one-third will require local treatment only and one-third will go on to require systemic treatment - the determinants of which include species type, number of lesions, immune status and the timing of treatment. The most common and widely studied systemic drugs are the antimony-based compounds (antimonials). The antileishmanial activity of antimonials has been known for nearly a century (11). Initially, this activity was discovered with compounds containing trivalent antimony (SbIII). However, the toxicity of these compounds precluded their widespread clinical use. Subsequently, therapy with compounds containing pentavalent antimony ( $\mathrm{SbV}$ ) was described in the treatment of cutaneous leishmaniasis in the 1940s (12) and, with little progress, remain the standard of care today. The prototypical drugs in this category are meglumine and sodium stibogluconate, of which only the latter is available in generic form and, hence, is usually the drug used in Latin America (13).

The mechanism of action of the antimony-based compounds is poorly understood (14). It is generally accepted that $\mathrm{SbV}$ is a prodrug that must be metabolized to SbIII to exert its antileishmanial effect. Pentavalent antimony can be converted into SbIII in the cytosol of the macrophage or within the amastigote itself. The mechanism of this conversion is debatable but may occur via a parasite-specific enzyme known as TDR1. Once the drug is converted into SbIII, it enters the amastigote by way of a membrane transporter called aquaglyceroporin (AQP1) (11).

Early experiments had suggested that SbIII inhibits energy metabolism and macromolecule synthesis in the parasite by decreasing the production of ATP via interference with glycolysis and the citric acid cycle (14). Subsequent studies have demonstrated that SbIII reduces the redox potential of the parasite by inducing the efflux of intracellular thiol compounds and inhibiting the enzyme trypanothione reductase, presumably leading to irreversible oxidative damage (15). In vitro studies in hematopoietic cell lines have shown that SbIII can inhibit certain protein tyrosine phosphatases as well (16).

A recent meta-analysis (13) demonstrated that the pentavalent antimonials are the most efficacious drugs available for treating cutaneous leishmaniasis. At a dose of $20 \mathrm{mg} / \mathrm{kg} /$ day for 20 days, the study found that $76.5 \%$ of patients treated with 
pentavalent antimonials experienced cure after completion of therapy. No significant differences were found between meglumine and stibogluconate in overall cure rates. Thus, the failure rate after therapy with pentavalent antimonials is $23.5 \%$. If initial therapy with pentavalent antimonials failed, a cure rate of $63.6 \%$ was found with repeat antimonial therapy.

The side effects of antimonial therapy can be substantial. In a year-long prospective study of 96 United States military personnel with confirmed leishmaniasis (17), the major side effects included arthralgias and myalgias (58\%), pancreatitis (97\%), transaminitis $(67 \%)$, headache $(22 \%)$, bone marrow suppression (44\%) and rash (9\%). Other side effects included nausea, vomiting, abdominal pain and reactivation of herpes zoster. Most side effects were reversible and abated within three to 12 days after initiation of therapy, without the need for discontinuation. Myalgias and arthralgias were reported for longer periods of time, but resolved with discontinuation of therapy, with a median duration of 20 days and 16 days, respectively (17).

Treatment was discontinued in $22 \%$ of the patients, with the most common cause for discontinuation being chemical pancreatitis (17). Treatment with stibogluconate was stopped if amylase levels became four times greater than the upper limit of normal or lipase levels became 15 times greater than the upper limit of normal. Three patients stopped treatment due to pancreatitis and one of these patients required parenteral hyperalimentation for one week. Discontinuation of therapy for all patients was temporary and was restarted within two to five days without further complications. At a median follow-up of 1.3 years after treatment with stibogluconate, none of the patients with cutaneous leishmaniasis developed visceral disease (17). Five patients (6\%) relapsed and were treated with a second course of stibogluconate, after which four of the five patients achieved clinical cure. The remaining patient with persistent disease was treated with a third course of stibogluconate and achieved clinical cure. No differences in cure rates were noted between those who had continuous or intermittent therapy (17).

Electrocardiographic changes were noted in $21 \%$ of patients and included flattening or inversion of $\mathrm{T}$ waves (17). A single HIV-positive patient treated with high-dose stibogluconate (1640 mg/day) for visceral disease developed a prolonged corrected QT interval without other identifiable causes such as electrolyte abnormalities. In a study (18) of 59 Kenyan patients with leishmaniasis who received one or more courses of sodium stibogluconate and were monitored with a baseline ECG and then serial ECGs throughout the course of treatment, ECG abnormalities were noted in 54\% of the treatment courses. The most common findings were flattening or inversion of $\mathrm{T}$ waves. Prolongation of the QT interval was noted in 13 patients, all of whom received stibogluconate at doses greater than $20 \mathrm{mg} / \mathrm{kg} /$ day or for more than 30 days (18). A total of three patients died during the study, and all three patients developed QT prolongation. However, two of these patients died of measles, and the third patient was receiving stibogluconate at three times the recommended dose (60 mg/kg/day) for four weeks (18). Given these significant toxicities, and their narrow therapeutic index, the use of antimonials often necessitates short-term hospitalization for patient monitoring, which compounds their cost and burden on the health care system.
For nonantimonial therapies, pentamidine had the highest therapeutic benefit (13). Cure rates with pentamidine were not significantly different compared with antimonials, although the point estimate of effect favoured the antimonials (OR 0.81; $95 \%$ CI 0.64 to 1.10 ). This, together with a larger body of clinical experience, makes antimonials the drugs of choice over pentamidine. Pentamidine has been shown to be more efficacious in the treatment of failures with antimonial therapy compared with repeat treatment with antimonials, with a cure rate of $87.2 \%$ versus $63.6 \%$. Thus, in the setting of treatment failure with antimonials, one may consider the use of pentamidine at a dose of $2 \mathrm{mg} / \mathrm{kg} /$ day to $4 \mathrm{mg} / \mathrm{kg} /$ day for three to eight days.

A 2009 Cochrane review (19) analyzed the outcomes of 38 trials involving 2728 patients with both cutaneous and mucocutaneous leishmaniasis. Systemic therapy with intravenous antimonials for 20 days was better than both three-day and seven-day antimonial regimens for infections with $L$ panamensis and L brasiliensis (19). Two trials demonstrated that the addition of oral allopurinol to intravenous antimonial therapy was superior to intravenous antimonial therapy alone in the treatment of $L$ panamensis and $L$ brasiliensis (RR 1.90; 95\% CI 1.40 to 2.59 ). In the treatment of $L$ brasiliensis infections in particular, there is evidence that the addition of oral pentoxifylline to intravenous sodium stibogluconate is superior to stibogluconate alone (RR 1.66; 95\% CI 1.03 to 2.69). In contrast with the findings of Tuon et al (13), the authors of the Cochrane review found that oral allopurinol was better than intravenous meglumine for the treatment of $L$ panamensis (RR 2.20; 95\% CI 1.34 to 3.60). Oral ketoconazole and miltefosine have also been shown to be better than placebo in $L$ panamensis infections, although miltefosine has less efficacy in $L$ brasiliensis and $L$ mexicana infections (10). Despite these results, the authors of the review conclude that larger and better conducted studies are required (19).

As with all vector-borne diseases, prevention is critical for travellers to endemic areas. Insect repellents and the regular use of long-sleeved shirts may reduce the risk of infection, although there are no specific data available with regards to optimal DEET concentrations (2). There is some evidence that impregnating clothing with permethrin reduces the risk of infection as well. A basic knowledge of the feeding habits of sandflies can be useful. Although most sandfly species are nocturnal feeders, those that carry parasites of the $L$ brasiliensis complex (most commonly Lutzomyia wellcomei) typically feed during the day, making these preventive measures especially important in the morning (2). Although fine-mesh bednetting is recommended for the prevention of mosquito bites, its usefulness in preventing sandfly bites is less clear because sandflies are smaller than mosquitos.

Despite the emergence of these New World diseases in industrialized countries, there is significant room for improvement with respect to making accurate diagnoses and implementing appropriate care. Delays in diagnosis and treatment often result from lack of disease recognition, but can also be attributed to delayed presentation. This dilemma is illustrated by this case nearly two years had passed between the initial presentation and the completion of antimonial treatment. Stibogluconate could only be obtained through Health Canada's Special Access Program after the patient failed initial therapy with itraconazole. 
His discharge from hospital was delayed until outpatient therapy could be arranged - a tedious process that required extensive multidisciplinary communication and collaboration.

This highlights the challenges patients and clinicians face in dealing with unfamiliar diseases in the returning traveller. These difficulties are compounded by the lack of research into these diseases. Diseases such as leishmaniasis, trachoma and leprosy typically affect the poorest populations of the world and are, thus, not lucrative and do not attract the research interest of large pharmaceutical companies $(6,20)$. Additionally, the infrastructure required for conducting clinical trials is not available in developing countries. Research of these diseases is also hindered by poor governance and the political oppression that often afflicts people in endemic regions.

As a result, physicians are compelled to use old and poorly investigated drugs that are difficult to access, and for which there are questionable safety and efficacy data. Patients are subjected to drugs with substantial toxicities that require in-hospital monitoring, with few alternatives. In this case, the lack of effective outpatient therapy necessitated the use of intravenous sodium stibogluconate therapy and short-term hospitalization. Given the costs of prolonged hospitalization and limited monitoring, failure rates with antimonial therapy are higher in studies

\section{REFERENCES}

1. Reithinger R, Dujardin JC, Louzir H, Pirmez C, Alexander B, Brooker S. Cutaneous leishmaniasis. Lancet Infect Dis 2007;7:581-96.

2. Schwartz E, Hatz C, Blum J. New world cutaneous leishmaniasis in travellers. Lancet Infect Dis 2006;6:342-9.

3. Statistics Canada <http://www40.statcan.gc.ca/101/cst01/arts37aeng.htm $>$ (Accessed on July 22, 2009).

4. Lawn SD, Whetham J, Chiodini PL, et al. New world mucosal and cutaneous leishmaniasis: An emerging health problem among British travellers. QJM 2004;97:781-8.

5. Zeegelaar JE, Steketee WH, van Thiel PP, Wetsteyn JC, Kager PA, Faber WR. Changing pattern of imported cutaneous leishmaniasis in the Netherlands. Clin Exp Dermatol 2005;30:1-5.

6. Beyrer C, Villar JC, Suwanvanichkij V, Singh S, Baral SD, Mills EJ. Neglected diseases, civil conflicts, and the right to health. Lancet 2007;370:619-27.

7. Freedman DO, Weld LH, Kozarsky PE, et al. Spectrum of disease and relation to place of exposure among ill returned travellers. New Engl J Med 2006;354:119-30.

8. Herwaldt BL. Leishmaniasis. Lancet 1999;354:1191-9.

9. Berman JD. Human leishmaniasis: Clinical, diagnostic, and chemotherapeutic developments in the last 10 years. Clin Infect Dis 1997;24:684-703.

10. Soto J, Arana BA, Toledo J, et al. Miltefosine for new world cutaneous leishmaniasis. Clin Infect Dis 2004;38:1266-72.

11. Ouellette M, Drummelsmith J, Papadopoulou B. Leishmaniasis: Drugs in the clinic, resistance and new developments. Drug Resist Updat 2004;7:257-66. performed in developing countries. There may be more pressure and incentive to conduct research as diseases such as leishmaniasis become more common in the developed world. We also advocate that medical schools in developed countries incorporate more education of tropical diseases in their curriculum, given the changing demographics and substantial increase in cross-border travel.

\section{CONCLUSION}

Cutaneous leishmaniasis is emerging as an important disease in the western world as travel to endemic regions becomes more common. The disease should be considered in the differential diagnosis of a dermatological disease in any traveller returning from Latin America. Accurate diagnosis and timely initiation of therapy are critical to the management of cutaneous leishmaniasis. The diagnostic method of choice is PCR analysis of a skin specimen for Leishmania DNA. Despite the high rate of failure and substantial toxicity, the pentavalent antimonials are firstline systemic therapy in the treatment of cutaneous leishmaniasis. Disease recognition and access to appropriate therapy are challenges in Canada, and the lack of research and development interest in diseases, such as cutaneous leishmaniasis, impedes progress in its treatment and negatively impacts patient care.

12. Templeton HJ. Cutaneous leishmaniasis, experimentally produced. Cal West Med 1941;54:70-1.

13. Tuon FF, Amato VS, Graf ME, Siqueira AM, Nicodemo AC, Neto VA. Treatment of new world cutaneous leishmaniasis a systematic review with a meta-analysis. Int J Dermatol 2008;47:109-24.

14. Berman JD, Waddell D, Hanson BD. Biochemical mechanisms of the antileishmanial activity of sodium stibogluconate. Antimicrob Agents Chemother 1985;27:916-20.

15. Wyllie S, Cunningham ML, Fairlamb AH. Dual action of antimonial drugs on thiol redox metabolism in the human pathogen Leishmania donovani. J Biol Chem 2004;279:39925-32.

16. Pathak MK, Yi T. Sodium stibogluconate is a potent inhibitor of protein tyrosine phosphatases and augments cytokine responses in hemopoietic cell lines. J Immunol 2001;167:3391-7.

17. Aronson NE, Wortmann GW, Johnson SC, et al. Safety and efficacy of sodium stibogluconate in the treatment of leishmaniasis: Recent U.S. military experience. Clin Infect Dis 1998;27:1457-64.

18. Chulay JC, Spencer HC, Mugambi M. Electrocardiographic changes during treatment of leishmaniasis with pentavalent antimony (sodium stibogluconate). Am J Trop Med Hyg 1985;43:702-9

19. Gonzalez U, Pinart M, Rengifo-Pardo M, Macaya A, Alvar J, Tweed JA. Interventions for American cutaneous and mucocutaneous leishmaniasis. Cochrane Database Syst Rev 2009;2:CD004834.

20. Chokshi DA, Rajkumar R. Leveraging university research to advance global health. JAMA 2007;298:1934-6. 


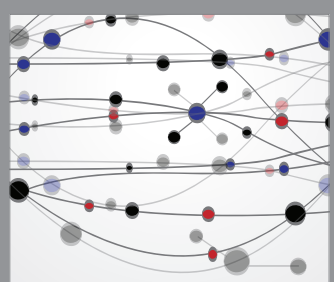

The Scientific World Journal
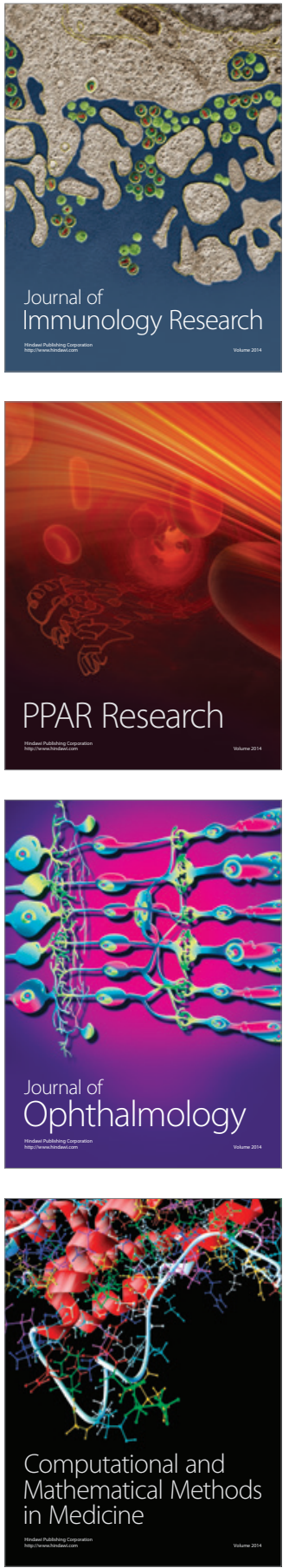

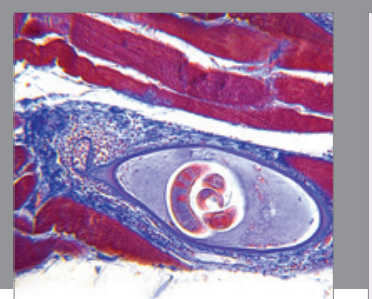

Gastroenterology Research and Practice

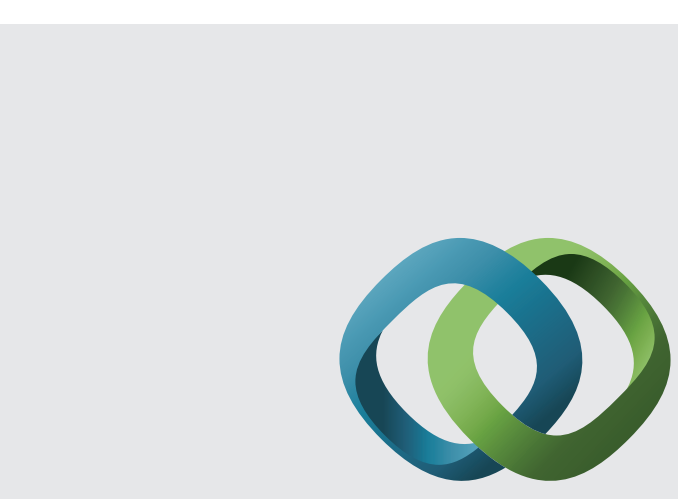

\section{Hindawi}

Submit your manuscripts at

http://www.hindawi.com
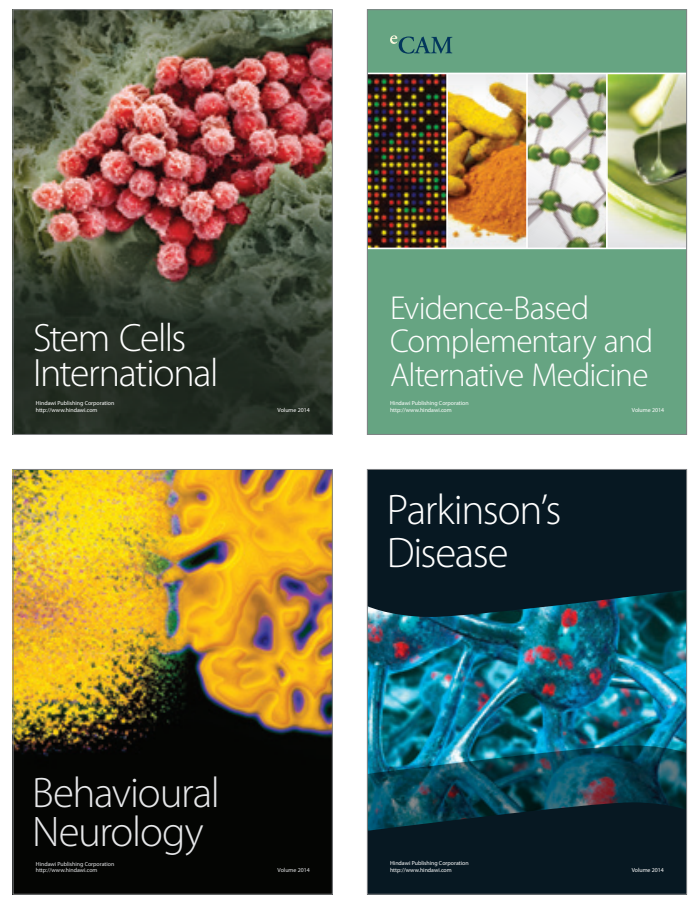
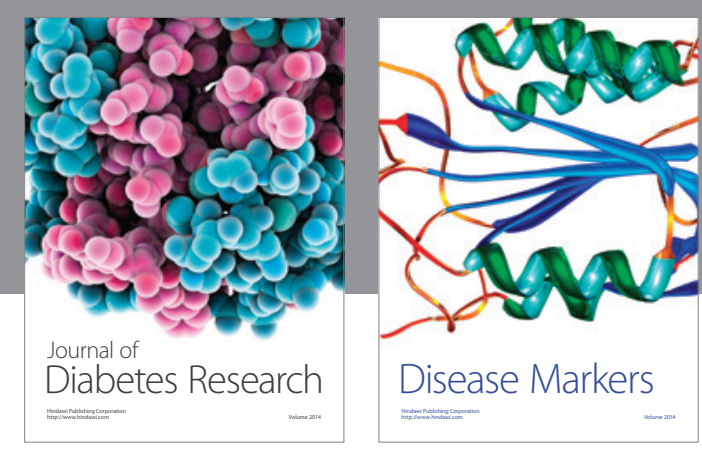

Disease Markers
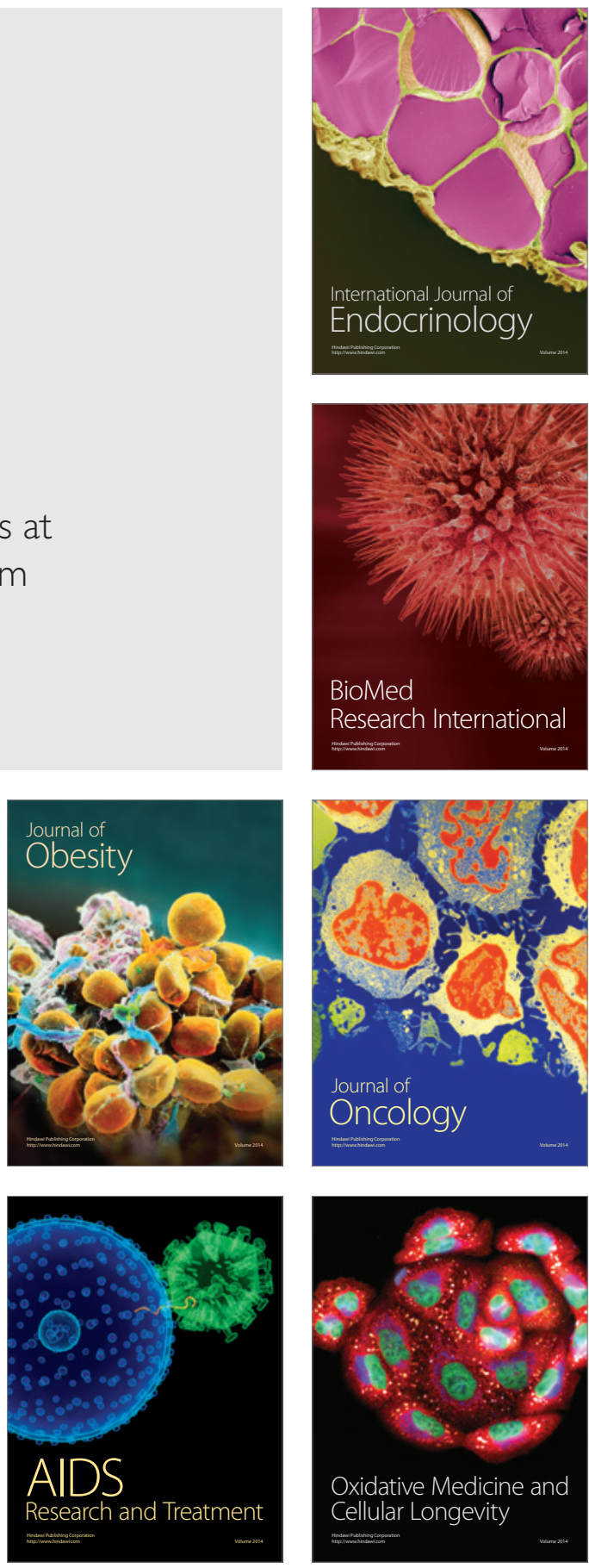